\title{
The Carcinogenic Agent Azoxymethane (AOM) Enhances Early Inflammation-induced Colon Crypt Pathology
}

\section{Freja Albjerg Venning ${ }^{1}$, Mogens Helweg Claesson ${ }^{1 *}$ and Hannelouise Kissow ${ }^{2}$}

${ }^{1}$ Department of International Health, Immunology and microbiology, Faculty of Health and Medical Sciences, University of Copenhagen, Copenhagen, Denmark ${ }^{2}$ Department of Biomedical Sciences, Faculty of Health and Medical Sciences, University of Copenhagen, Copenhagen, Denmark

\begin{abstract}
Severe combined immunodeficiency (SCID) mice transplanted with CD4+ T cells depleted of CD25+ regulatory T cells develop colitis within 2-3 weeks after the T cell transfer. In the present study we studied the effect of the carcinogen azoxymethane (AOM) on the colon crypt pathology of normal SCID mice and SCID mice with transfer colitis. AOM itself did not result in neither weight loss nor inflammation although treatment affected crypt widths and numbers. Although AOM together with T cell transfer did not increase the level of gut inflammation including COX-2 expression, AOM increased crypt changes associated with colon inflammation such as a decline in crypt numbers and an increase in crypts width throughout the large intestine. Thus it appears that AOM lower the threshold level for inflammation-induced changes which potentially may lead to neoplasia.
\end{abstract}

Keywords: Neoplasia; Carcinogenic agent azoxymethane; Histopathology

\section{Introduction}

It is generally accepted that chronic inflammation play an important role in development of neoplasia and some cellular and molecular mechanisms responsible for transition to neoplasia have been elucidated [1-5]. Around 20\% of human cancers may be caused by chronic inflammation including gastric ulcer (helicobacter pylori), cervix carcinoma (human papilloma virus), chronic hepatitis (hepatitis virus $\mathrm{B}$ and $\mathrm{C}$ ) and chronic parasite infections (leismania mansoni) [6]. In humans, inflammatory bowel diseases (IBD, ulcerative colitis and Crohn's disease) are uncontrolled immune reactivity against the intestinal micro flora which is the driving force for these chronic inflammations and chronic inflammation per se may increase the risk for colorectal carcinoma [7-9].

In the present work we have employed a mouse model for chronic inflammation the so called $\mathrm{T}$ cell transfer colitis [10-12]. This form of colitis has in contrast to chemically and dextran sulfate (DS) induced colitis many resemblances to IBD in humans at the immunopathological as well as at the molecular level [13]. The carcinogenic agent azoxymethane (AOM) has been used because the resulting carcinogenesis shows certain similarities with spontaneously arising colorectal carcinoma in humans [14]. However, in order to initiate colon carcinoma with single doses of AOM, the colon needs an inflammatory stimulus causing chronic inflammation $[15,16]$. In the present work, colitis was induced in SCID mice by transfer of CD4+ $\mathrm{T}$ cell depleted of T reg cells $[11,12,17]$ and $\mathrm{AOM}$ was supplied as two single doses within the first week after $\mathrm{T}$ cell transfer. This treatment scenario mimics the human conditions where patients with IBD subsequently may be exposed to genotoxic agents in the diet causing DNA damage eventually leading to colorectal cancer.

\section{Materials and Methods}

\section{Induction of colitis}

Six-week-old female SCID mice and BALB/c mice were purchased from Bomholtgaard, Ry, Denmark. SCID mice are homozygous for the scid mutation, and lack mature T and B lymphocytes. Mice were kept in a controlled microbial environment in individually ventilated cages with 8 mice per cage at the Department of Experimental
Medicine at the Panum Institute. The animal studies were approved by the Danish National Committee for Animal Studies. The mice were nourished throughout the study with water and lab chow (no. 1314, Altromin, Lage, Germany) ad libitum. Mice were randomized into four groups; TRANS, TRANS/AOM, AOM and CONTROL. Colitis was induced in the TRANS and the TRANS/AOM group of SCID mice by transplantation of $\mathrm{CD} 4+\mathrm{CD} 25-\mathrm{T}$ cells (devoid of $\mathrm{T}$ regs) from the mesenteric lymph nodes of normal BALB/c mice as described previously $[11,12,17]$. In brief, $\mathrm{CD} 4^{+} \mathrm{T}$ cells were isolated directly from single cell suspensions of with anti-CD4 Dynabeads and Detach-aBead (Dynal A/S, Norway) according to the manufacture's protocol. The isolated CD4 ${ }^{+} \mathrm{T}$ cells were then depleted of CD25+ T and 300,000 cells were injected i.p. The AOM and CONTROL group received a saline injection i.p. The day after transplantation the TRANS/AOM and the AOM group received an injection i.p. with azoxymethane $5 \mathrm{mg} / \mathrm{kg}$ body weight. This was repeated at day 7. The TRANS and CONTROL group received a saline injection i.p. AOM at single doses of $10 \mathrm{mg} /$ $\mathrm{kg}$ body weight used in previous work $[15,16]$, proved to be toxic and killed most of our 6 week old recipient SCID mice within $24 \mathrm{hrs}$ after injection.

All animals were monitored daily for signs of intestinal dysfunction such as cachexia, diarrhea, bloody stools, piloerection and rectal prolapsed, and body weight were recorded every second day. At 25, 39 and 53 days after transplantation 3-4 mice from each group were anaesthetized with ketamine/xylazine $100 / 10 \mathrm{mg} / \mathrm{kg}$ i.p. (Pharmacy Service, LIFE, University of Copenhagen, Denmark), the animals were weighed and the abdomens were opened. To smooth the mucosal folds, the colon was primarily fixed for 3-5 minutes in situ by intra-rectal

*Corresponding author: Mogens $\mathrm{H}$ Claesson, Professor, DMSc, The Panum Institute, Blegdamsvej 3, 2200 Copenhagen, Denmark, Tel: 45 35327270; Fax: 45 35327269; E-mail: claesson@sund.ku.dk

Received August 31, 2013; Accepted October 24, 2013; Published October 28, 2013

Citation: Venning FA, Claesson MH, Kissow H (2013) The Carcinogenic Agent Azoxymethane (AOM) Enhances Early Inflammation-induced Colon Crypt Pathology. J Cancer Sci Ther 5: 377-383. doi:10.4172/1948-5956.1000229

Copyright: (c) 2013 Venning FA, et al. This is an open-access article distributed under the terms of the Creative Commons Attribution License, which permits unrestricted use, distribution, and reproduction in any medium, provided the original author and source are credited. 
infusion of $4 \%$ paraformaldehyde. The colon was then removed, cut open, pinned to a polyethylene plate and stored for an additional 24 hours in paraformaldehyde and thereafter in $70 \%$ ethanol until analysis.

\section{Macroscopic analysis of the colon}

The colon specimens were stained as whole mounts with $0.2 \%$ methylene blue for 1 minute according to Bird et al. [18] and examined under a stereomicroscope (Bresser Advance ICD, Meade Instruments Europe, Rhede, Germany). Pictures covering $0.5 \mathrm{~cm}^{2}$ the luminal surface were taken every $2 \mathrm{~cm}$ starting from the ileocecal junction (Bresser MicroCam 3.0, Meade Instruments Europe, Rhede, Germany) and analyzed with the ImagePro software program. (ImagePro 7.0 software Media Cybernetics, Inc. Bethesda, MD, USA). We examined the number of crypts per $\mathrm{mm}^{2}$, by counting crypt number in a grid of $1 \mathrm{~mm}^{2}$, four times in each picture. We estimated the crypt width by measuring approximately 100 random crypts in each picture.

After pictures were taken, we cut out specimens of $2 \mathrm{~mm}^{2}$ in the rectum and mid colon. To visualize an eventually mucin-depletion and small adenomas the colons were stained using the High Iron DiamineAlcian High Iron Diamine-Alcian Blue (HID-AB) procedure described in [19].

\section{Histology and immunohistochemistry}

Tissue was embedded in paraffin and cut into $5 \mu \mathrm{m}$ sections, and stained with hematoxylin/PAS.

Histopathology scoring was performed blindly according to previously published criteria [10-12]: Mild colitis: crypt enlargement, increased mononuclear cell infiltration in the colonic lamina propria (score 1); moderate colitis: enlargement of crypts with increased mitotic activity and goblet cell attenuation besides mononuclear cell infiltration (score 2-3); severe colitis: infiltration of lamina propria and submucosa with mononuclear and polymorphonuclear cells, crypt hyperproliferation and destruction (score 4-5). In the histological sections from mid colon and rectum crypt depth was measured using the Image Pro software (Media Cybernetics, Inc. Bethesda, MD, USA).

For immunhistochemistry [20] antigen retrieval was performed by heating the sections for 15 minutes in a microwave oven $(750 \mathrm{~W})$ in citrate buffer $\mathrm{pH}$ 6.0. The sections were then incubated for 10 minutes in $2 \%$ bovine serum albumin, followed by overnight incubation with anti human COX 2 monoclonal antibody (Clone SP21, Nordic Biosite, Denmark) diluted 1:200. For detection and visualization of the immunoreactions the sections were incubated for 40 minutes with biotinylated goat anti-rabbit IgG (Vector BA-1000) diluted 1:200 (Vector Laboratories, Burlingame, CA) followed by StreptABComplex/ horseradish peroxidase (Vectastain ABC kit, Vector Laboratories, Burlingame, CA). Finally the reaction was developed by the use of 3,3diaminobenzidine (KEM-EN-TEC, cat.no. 4170) for 15 min., followed by $2 \mathrm{~min}$. incubation in $0.5 \%$ Copper sulphate (Merck art.no. 2790) diluted in Tris buffer containing $0.05 \%$ Tween. Counterstaining was performed with Mayers Hemalun.

Presence of COX-2 immuno-positive crypt cells was counted manually.

\section{Statistic analysis}

All results are shown as means \pm SEM. Comparison between the four groups was performed with Two-way ANOVA, followed by comparisons of the each group using Bonferroni's Test. All analyses were performed using GraphPad Prism version 5.01

\section{Results}

\section{Body weights}

Figure 1 shows the changes in body weights after AOM treatment, after transfer of $\mathrm{CD} 4+\mathrm{CD} 25-\mathrm{T}$ cells, and after $\mathrm{AOM}$ treatment combined with transfer of $\mathrm{CD} 4+\mathrm{CD} 25-\mathrm{T}$ cells $(\mathrm{AOM}$, TRANS,TRANS/AOM groups). The figure shows body weights of mice throughout the experimental period (Figure 1A). Although all transplanted mice showed clinical (and histopathological) signs of colitis such as sticky feces, diarrhea and inflammation (see below) from day 25 onwards, the body weight of the transplanted group first started to deviate from the non-transplanted-AOM treated group during the last 10 days of the experimental period. From day 7 to 39 the TRANS/ AOM group showed more severe body weight loss than mice in the TRANS group $(\mathrm{p}<0.001$; ANOVA two-sided test). However, from day 42 onwards the body weights were identical in these two groups of mice. AOM treatment alone did not lead to loss of body weight when compared with the weights of 6 weeks old healthy SCID mice tested in two separate experiments (Figure 1 B). At day 25, 39 and 53 groups of individual mice were sacrificed and the large intestine removed for mucosa examinations.

\section{Qualitative assessment of the luminal colon surface}

The luminal surface of the untreated SCID mice appeared normal, with no objective changes from what we usually see in normal BALB/c mice. Colon surface from AOM treated mice also showed a near normal surface, with normal crypts, but with a few aberrant crypts or aberrant crypts foci (ACF) as visualized in Figure 2G.

The number of ACF varied from 4 to 16 and there was a tendency to an increase with time after AOM injection, but this did not reach statistical difference (results not shown). When we looked at the intestine from the TRANS and the TRANS/AOM group we found a severely inflamed surface. The crypts were enlarged and stained darker with methylene blue. There were fewer crypts, with space in between. Comparing these with the AOM group it appeared if all the crypts were aberrant, and none of the crypts were normal size. We also found areas with total crypt loss and crypt disruption, and several small ulcerations. Objectively the colons from the AOM/TRANS grouped appeared to have larger and fewer crypts than the colons from the TRANS group; therefore we counted the number of crypts $/ \mathrm{mm}^{2}$ and measured the crypt width.

To investigate if these changes were due to a depletion of mucin we stained the intestine with HID-AB, which stains the sulphomucins and sialomucins. We did not find any areas with total mucin depletion, in any of the groups, so this staining did not gain any new information.

\section{Crypt widths and numbers}

Mounted parformaldehyde fixed whole large intestine preparations were stained with methylene blue and the crypt surface morphology was examined in a stereomicroscope (Figure 2). The AOM and control groups show similar (normal) crypt morphology whereas the majority of the crypts of the TRANS and TRANS/AOM groups show much larger crypt widths than the two former groups. On the basis of surface microscopy, the number of crypts and crypt widths throughout the large intestine were evaluated; origins of the colons were blinded for the investigator.

The changes in crypt numbers per $\mathrm{mm}^{2}$ large intestine in SCID mice 25, 39 and 53 days after T-cell transfer are shown in Figure 3A-3F. 

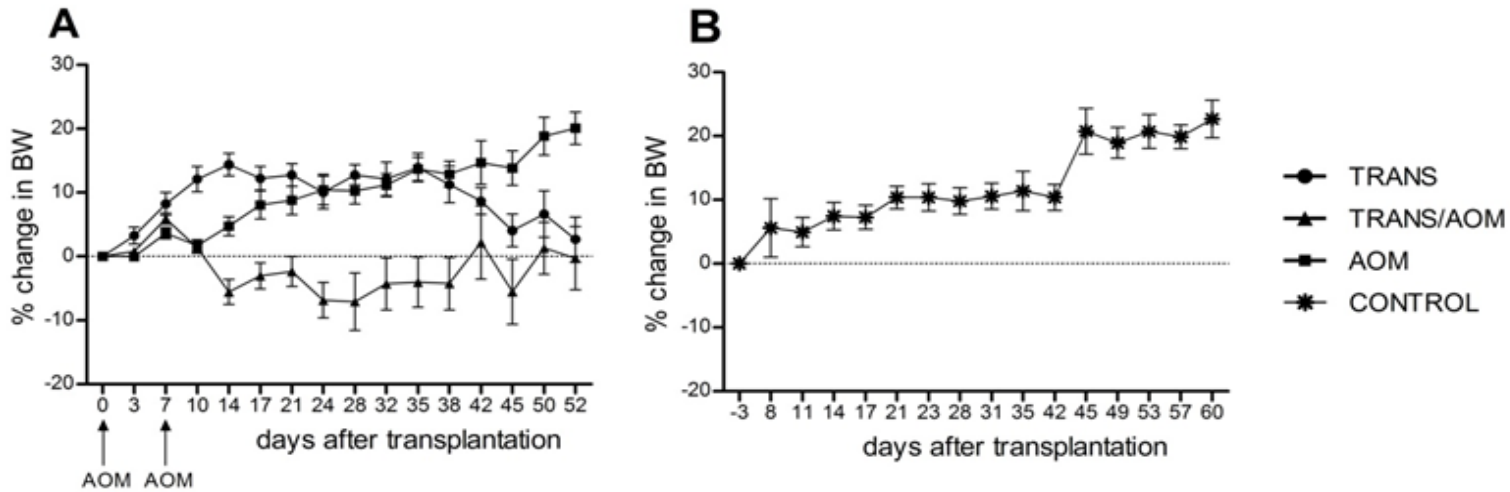

Figure1: Normalized body weight change in all mice, four mice from each group was euthanized at day 25,39 and 53 (A). Normalized body weight changes over time for healthy young adult SCID mice (B).

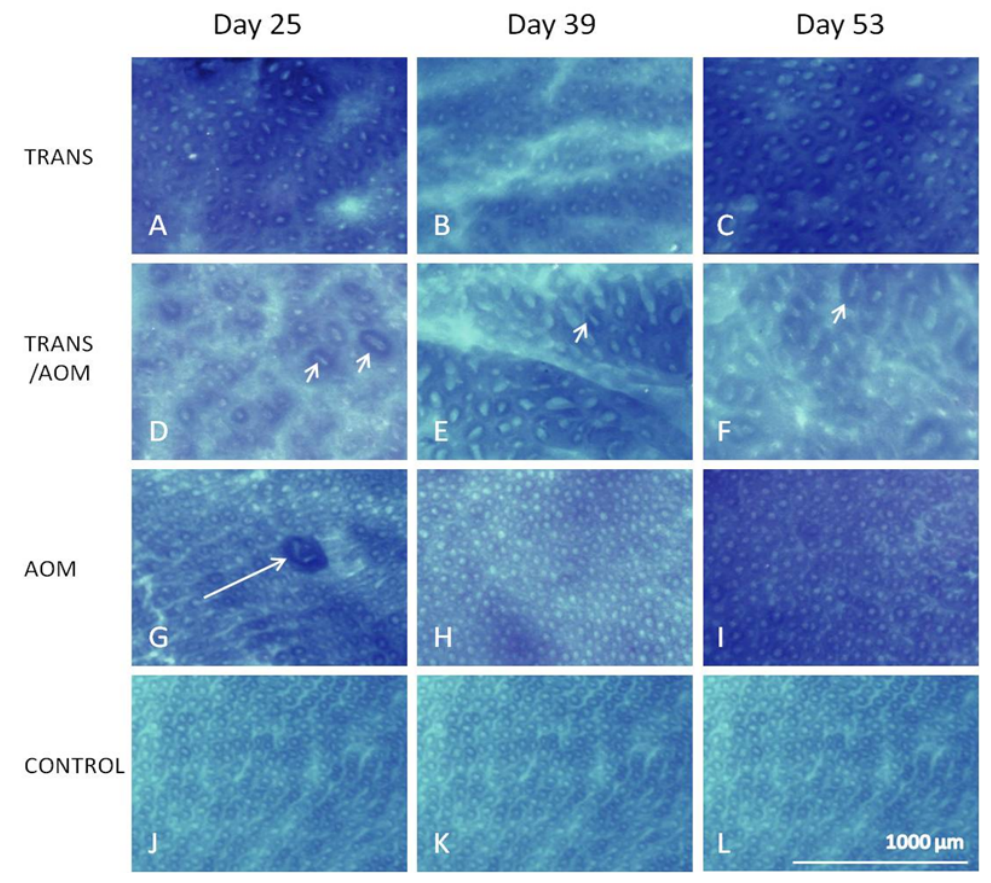

Figure 2: Examples of crypt widths of individual colons from the TRANS. TRANS/AOM, AOM and control groups. Note that the AOM and control groups show similar crypt morphology whereas the majority of the crypts of the TRANS and TRANS/AOM groups show much larger crypt widths than the two former groups. Occasionally, aberrant crypt foci (ACF) were observed in AOM treated mice (arrow). Many crypts in the TRANS and TRANS/AOM remind of ACF (arrow heads).

The large intestine was examined from the ileocecal junction (prox) to the anus at intervals of $2 \mathrm{~cm}$. At the three post transplantation time intervals, $\mathrm{A}, \mathrm{B}$ and $\mathrm{C}$ respectively, the TRANS and the TRANS/ AOM groups showed the same significantly lower numbers of crypt/ $\mathrm{mm}^{2}$ compared with the AOM and the control group, respectively. In addition, the AOM group showed significantly fewer crypts than the control group at 53 days post $\mathrm{T}$ cell transfer. Figure $3 \mathrm{D}-\mathrm{F}$ shows the total numbers of crypts in the large intestine of the four groups of mice at the three time intervals of observation. As above, the TRANS and the TRANS/AOM groups showed very significant decreased numbers of crypts compared with the control group. Also, the TRANS/AOM at 38 and 53 days post $\mathrm{T}$ cell transfer showed significantly fewer crypts compared with the TRANS group. At all time intervals the control group tended to have higher crypt numbers than the AOM group (see statistics in the figure legends).

Figure $4 \mathrm{~A}-4 \mathrm{~F}$ shows the quantitative changes in the crypt width for the TRANS, TRANS/AOM, AOM and control groups, respectively, throughout the large intestine and as a function of time after $\mathrm{T}$ cell transfer. The TRANS and TRANS/AOM showed increased crypt width compared with the AOM and the control groups. Particularly in the distal colon at 25 and 53 days after T cell transfer the TRANS/AOM group showed increased crypt widths compared with the three other groups. Figure $4 \mathrm{D}-4 \mathrm{~F}$ shows crypt widths for the entire large intestine of the four groups of mice at the three time intervals of observation. At all time intervals, the TRANS and the TRANS/AOM groups showed increased crypt widths compared with the AOM and the control 

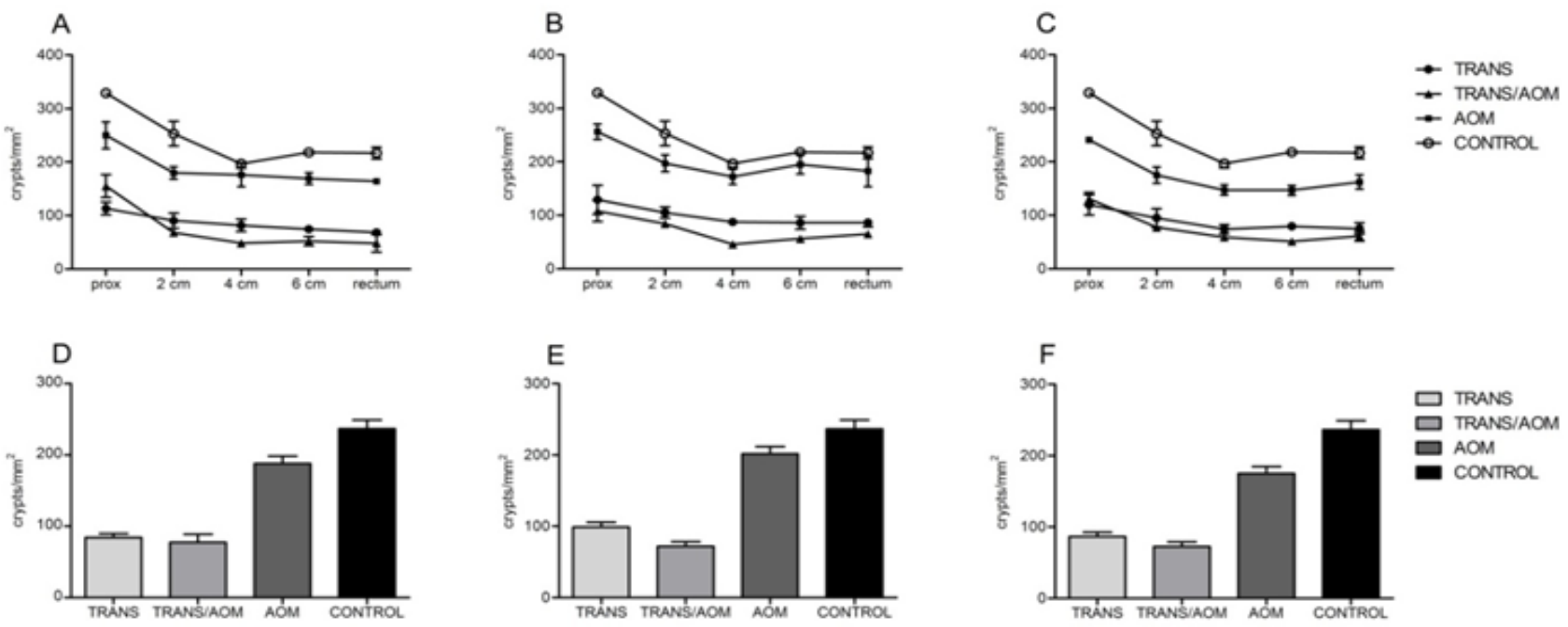

Figure 3: Changes in crypt numbers per $\mathrm{mm}^{2}$ large intestine in SCID mice at 25 (A,D), 39 (B,E) and 53 (C,F) days after T cell transfer. The large intestine was examined from the ileocecal junction (prox) to the anus at intervals of $2 \mathrm{~cm}$. A,B,C: TRANS < CONTROL, $p<0.01$; TRANS/AOM $<A O M$ and CONTROL, $p<0.01$. C: CONTROL > AOM, $p<0.05 ; D, E, F:$ TRANS and TRANS/AOM<AOM and CONTROL, $p<0.01 ; A$ OM $<$ AOM and CONTROL, $p<0.01 ; E:$ TRANS/AOM < TRANS, $p<0.05$.
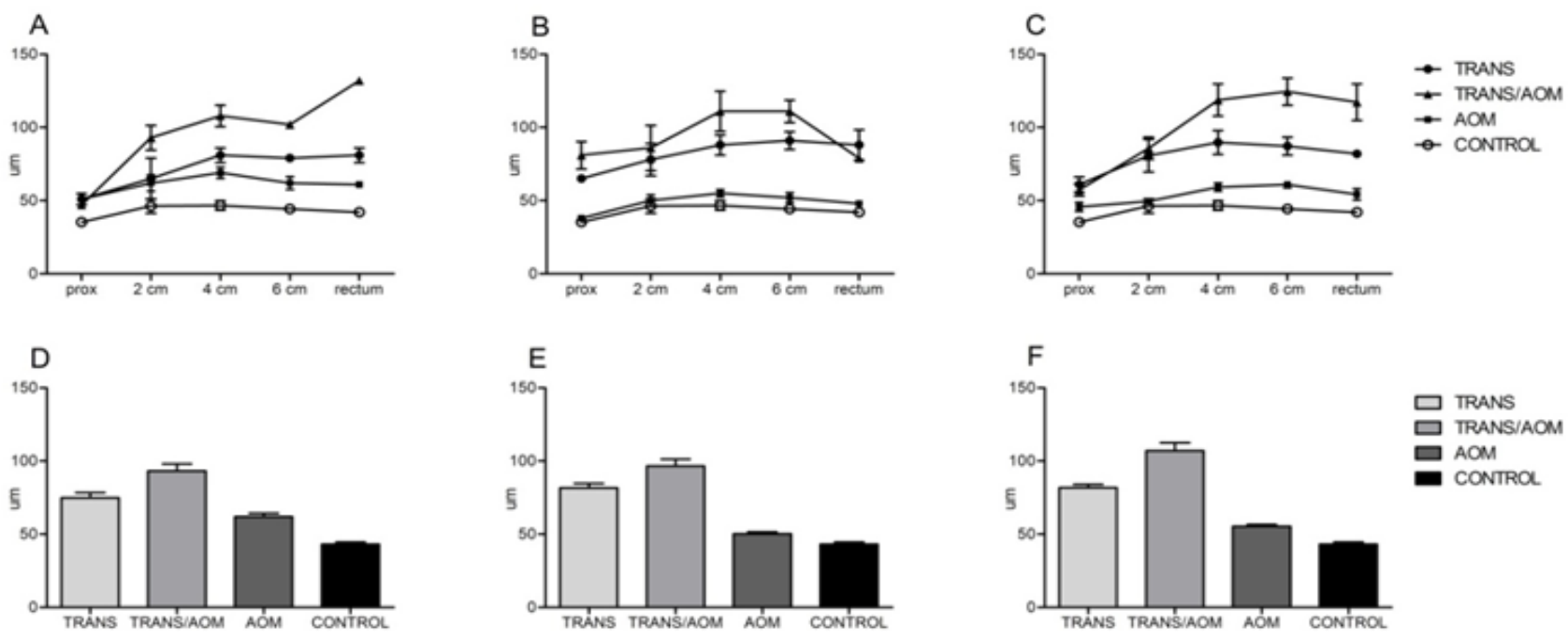

Figure 4: Crypt widths in the large intestine in SCID mice at $25(A, D), 39(B, E)$ and $53(C, F)$ days after T cell transfer. The large intestine was examined from the ileocecal junction (prox) to the anus at intervals of $2 \mathrm{~cm}$. A,B,C: TRANS and TRANS/AOM < CONTROL, $p<0.01$; $B$ : TRANS/AOM < TRANS, p<0.05; B,C: AOM < CONTROL, $p<0.05$. D,E,F: TRANS and TRANS/AOM > CONTROL, $p<0.01$; TRANS/AOM > TRANS, $p<0.01$. D: AOM > CONTROL, $p<0.05$.

groups. The TRANS/AOM group in showed crypt widths significantly above those of the TRANS group (see statistics in the figure legends).

Thus AOM treatment with or without colitis reduced the total numbers of colonic crypts and lead to an overall increased crypt width.

\section{Colitis score and crypt depth}

Individual samples from mid colon and rectum were scored for crypt depth, signs of crypt degeneration, crypt abscesses, cellular infiltration in sub-epithelial and sub-mucosal layers and the occurrence of ulcers. The colitis scores (mean score from rectum and mid colon) are depicted in Figure 5A. Neither control mice nor the AOM group showed signs of inflammation. The TRANS and the TRANS/AOM groups showed comparable levels of inflammation throughout the observation period. The crypt depth increased equally in the TRANS and TRANS/AOM group compared to AOM and CONTROL (Figure $5 \mathrm{~B}$ ) as a sign of hyper-proliferation, but this was equal in the two groups. Figure $5 \mathrm{C}$ shows the rectum histopathology of the four groups of mice.

\section{COX-2 expression}

Increased COX-2 activity, an enzyme responsible for the formation of prostanoids, may be involved in the progression of colorectal cancer [21]. Here we studied if the combination of colitis and AOM treatment 
Citation: Venning FA, Claesson MH, Kissow H (2013) The Carcinogenic Agent Azoxymethane (AOM) Enhances Early Inflammation-induced Colon Crypt Pathology. J Cancer Sci Ther 5: 377-383. doi:10.4172/1948-5956.1000229

changed the expression of COX-2 in the colon epithelium. Counting the number of colon crypts being positive for COX-2 immunostaining showed that none of the crypts in mice from the AOM or CONTROL groups were positive. In contrast, mice from the TRANS and TRANS/ AOM groups having a colitis score above 2.5 had $50-95 \%$ COX-2 positive crypts regardless of treatment. Thus colitis per se is associated with significant epithelial expression of COX-2 which is not affected by simultaneous AOM treatment (Figure 6).

\section{Discussion}

Crypts with an abnormal morphology in colon and rectum such as aberrant crypt foci (ACF) and mucin depleted foci (MDF) are considered to be pre-malignant lesions identified in carcinogen treated rodents and humans [22,23]. In the present work we studied if the carcinogen AOM influenced the morphology and numbers of crypts in the large intestine of SCID mice in the course of $\mathrm{T}$ cell transfer colitis. Colitis was induced in SCID mice by transfer of T reg depleted CD4+CD25- T cells $[11,12,17]$.

Surprisingly, the early change in body weights after transfer of CD4+CD25- T cells [10-12] did not occur in the present experiment although the mice showed clinical and histopathological signs of colitis from day 25 onwards. This might be due to the strictly controlled microbial environment in our new animal facility with individually ventilated cages (see M\&M section). However, SCID mice receiving both AOM and $\mathrm{T}$ cell transfer showed an initial weight loss, which might reflect the toxic effect of AOM (see below). In contrast, mice treated with $\mathrm{AOM}$ alone gained weight at a rate comparable with that of normal 6 weeks old SCID mice. Thus it appears that it is the combined effect of colitis-inducing T cells and AOM which increases the toxicity of the latter agent.

Because SCID mice suffer from a genetic defect of the gene
A

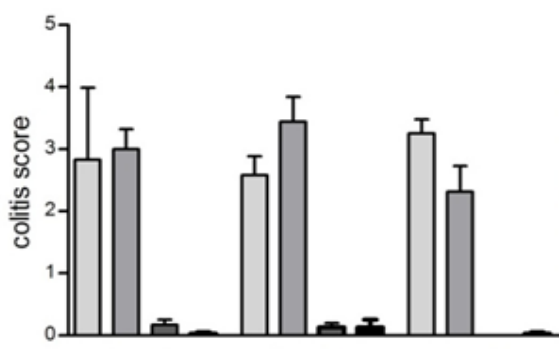

day 25 day 39

B

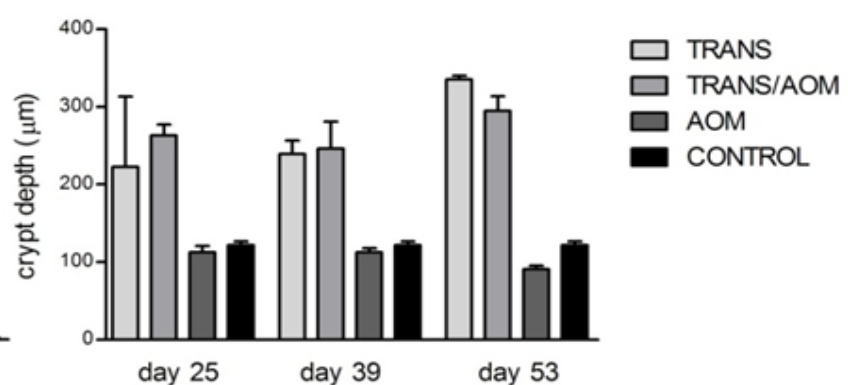

$c$

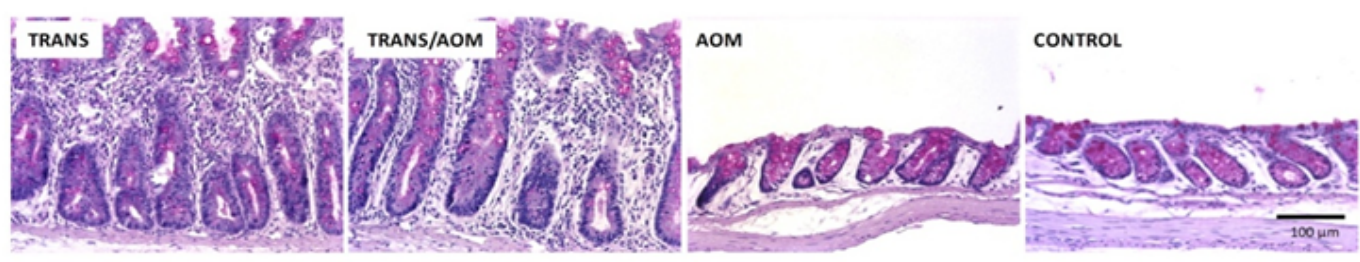

Figure 5: Colitis score values from SCID mice at day 25, 39 and 53 after transplantation showing colitis score for the TRANS, TRANS/AOM, AOM groups and a nontreated control group. Although some fluctuations in colitis score appeared for the TRANS and the TRANS/AOM groups during the period, these were not significant for any particular day or treatment (A). Mean crypt depth in sections from mid colon and rectum. Crypts in the TRANS and TRANS/AOM group were hyper-proliferative with a crypt depth more than two fold of crypts from a non-treated control group. No difference between TRANS and TRANS/AOM (B). Photographs showing similar degrees of inflammation in the TRANS and TRANS/AOM groups and the absence of inflammation in AOM treated mice (C).
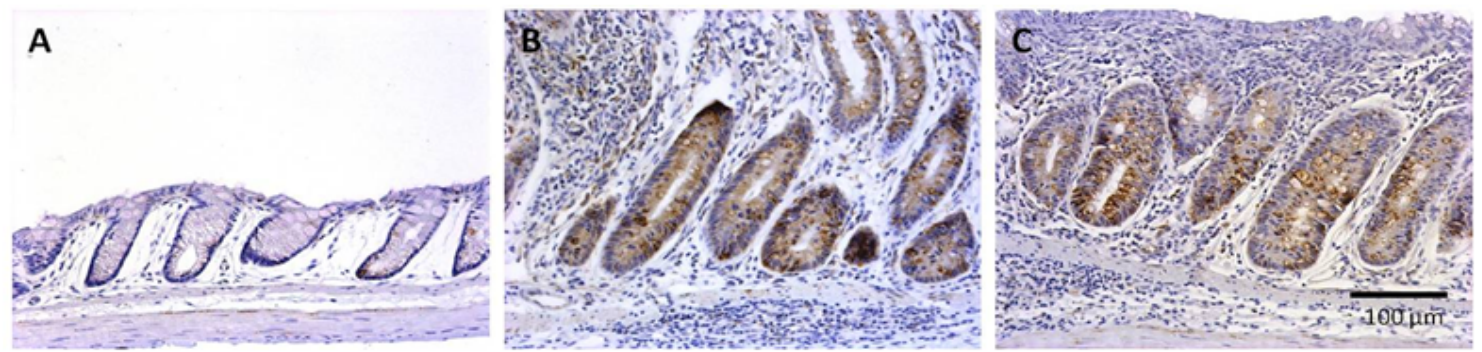

Figure 6: Examples of Cox-2 immunostaining in AOM (A), TRANS (B) and TRANS/AOM (C). No differences in number of positive crypts were found between AOM and CONTROL or between TRANS and TRANS/AOM groups. 
encoding a catalytic subunit of DNA dependent protein kinase [24], involved in transcription of the $\mathrm{T}$ cell receptor and immunoglobulin genes [25], SCID mice are born without a functioning cellular and humoral adaptive immune system [26]. Lack of adaptive immunity may render SCID mice more susceptible to the effects of carcinogens as indicated previously [27] showing that two weekly AOM treatments of SCID mice already at 4 weeks resulted in development of a significant higher number of ACF per colon compared with ACF development in congenic normal CB-17 control mice treated with AOM. Six weeks of AOM treatment resulted in occurrence of adenocarcinomas in SCID mice already after 100 days, 20 days prior to the development of carcinomas in the control mice [27]. In our material we did not observe development of adenomas or adenocarcinomas neither in the AOM nor in the TRANS/AOM groups. The dose of AOM, $5 \mathrm{mg} / \mathrm{kg}$ body weight injected ip. on two consecutive weeks, used in the present study, is only $50 \%$ of the dose used by Ochiai et al. [27] and might explain the less severe changes observed here compared with that study. However, AOM at $10 \mathrm{mg} / \mathrm{kg}$ body weight proved to be toxic for our six week old female SCID mice and killed most of the animals within $24 \mathrm{hrs}$ after injection. Also, due to the devastating effect of colitis on the general health of the mice, we had to terminate the experiments after 53 days which is too early to expect neoplastic changes [16,27]. Occasionally, we observed occurrence of typical ACs or ACF after AOM treatment (Figure $3 \mathrm{G}$ ). The ACs induced by AOM alone were few, darkly stained, were either solely or clustered in foci and were different from the surrounding colonic crypts as described by Bird et al. [18]. In the TRANS and TRANS/AOM groups all colonic crypts had the characteristic changes of an ACs as described by Bird et al. [18] but they were not clustered in foci. The crypts were significantly wider and fewer per area than the crypts in the AOM and CONTROL groups. The morphology of these crypts closely resembles but is not identical with typical ACF. Typical ACF could not be localized in T cell transplanted colitis SCID mice neither in non-AOM treated nor in AOM treated mice. In the TRANS/AOM group, however, both crypt numbers and crypt widths were more severely affected than those observed in the TRANS group, suggesting that the characteristic changes of the colonic crypts seen in $\mathrm{T}$ cell transfer colitis were exaggerated by treatment with the carcinogen AOM.

AOM treatment combined with $\mathrm{T}$ cell transfer resulted in an accelerated body weight loss compared with the TRANS group. Since body weight loss in $\mathrm{T}$ cell transfer colitis correlates with the severity of the disease, the inflammation in the TRANS/AOM group might initially be more severe than in the TRANS group. However, colitis score measurements including the level of COX-2 expression did not differ between the TRANS and the TRANS/AOM groups.

It is generally accepted that crypt abnormalities such as ACF are pre-cancerous lesions [28]. Although the crypt abnormalities in transfer colitis did not develop into typical ACF the fact that a carcinogenic agent such as AOM enhances the abnormal crypts, might suggest that, likewise ACF, crypts with increased widths in colitis mice may reflect putative pre-malignant changes.

\section{Conclusion}

Taken together our data demonstrate that CD4+CD25- $\mathrm{T}$ cell transfer to SCID mice leads to gut inflammation associated with a decreased number of crypts and an increased crypt width throughout the large intestine. Whereas gut inflammation including COX-2 expression is unaffected by AOM treatment, colitis-induced crypt changes are further increased in the presence of AOM treatment.
Likewise, AOM treatment alone results in minor although recognizable crypt pathology. These data strongly suggest that carcinogens like AOM, having only minor influence on early colon pathology by itself, has an enhancing effect on the colon pathology at sites of T cell-induced inflammation, which could be extrapolated to a possible enhancing effects of environmental carcinogens on various inflammatory diseases. We hypothesize that pathological changes such as an increased crypt width and reduction in crypt numbers may be the first step leading to aberrant crypt formation, a development which may precede neoplastic lesions [22,28]. The present observations of carcinogen-induced early changes in the inflammatory gut mucosa might thus reflect early stages of lesions with a neoplastic potential.

\section{Acknowledgements}

This work was supported by Poul Riis and Vibeke Binder Foundation, Hede Nielsen Foundation and Wedell Wedellsborg Foundation. The authors thank Heidi Paulsen for technical assistance.

\section{Disclosure statement}

FAV, MHC and HK have nothing to declare.

\section{References}

1. Terzic J, Grivennikov S, Karin E, Karin M (2010) Inflammation and colon cancer Gastroenterology 138: 2101-2114.

2. Raman D, Baugher PJ, Thu YM, Richmond A (2007) Role of chemokines in tumor growth. Cancer Lett 256: 137-165.

3. Ostrand-Rosenberg S (2008) Immune surveillance: a balance between protumor and antitumor immunity. Curr Opin Genet Dev 18: 11-18.

4. Mantovani A, Allavena P, Sica A, Balkwill F (2008) Cancer-related inflammation Nature 454: 436-444

5. Hong S, Lee HJ, Kim SJ, Hahm KB (2010) Connection between inflammation and carcinogenesis in gastrointestinal tract: focus on TGF-beta signaling World J Gastroenterol 16: 2080-2093.

6. Rakoff-Nahoum S (2006) Why cancer and inflammation? Yale J Biol Med 79 123-130.

7. Eaden JA, Abrams KR, Mayberry JF (2001) The risk of colorectal cancer in ulcerative colitis: a meta-analysis. Gut 48: 526-535.

8. Ekbom A, Helmick C, Zack M, Adami HO (1990) Ulcerative colitis and colorectal cancer. A population-based study. N Engl J Med 323: 1228-1233.

9. Rutter MD, Saunders BP, Wilkinson KH, Rumbles S, Schofield G, et al. (2006) Thirty-year analysis of a colonoscopic surveillance program for neoplasia in ulcerative colitis. Gastroenterology 130: 1030-1038.

10. Claesson $\mathrm{MH}$, Bregenholt S, Bonhagen K, Thoma S, Möller P, et al. (1999) Colitis-inducing potency of CD4+ T cells in immunodeficient, adoptive hosts depends on their state of activation, IL-12 responsiveness, and CD45RB surface phenotype. J Immunol 162: 3702-3710.

11. Gad M, Kristensen NN, Kury E, Claesson MH (2004) Characterization of T-regulatory cells, induced by immature dendritic cells, which inhibit enteroantigen-reactive colitis-inducing T-cell responses in vitro and in vivo. Immunology 113: 499-508.

12. Kristensen NN, Gad M, Thomsen AR, Lu B, Gerard C, et al. (2006) CXC chemokine receptor 3 expression increases the disease-inducing potential of CD4+ CD25- T cells in adoptive transfer colitis. Inflamm Bowel Dis 12: 374-381

13. te Velde AA, de Kort F, Sterrenburg E, Pronk I, ten Kate FJ, et al. (2007) Comparative analysis of colonic gene expression of three experimental colitis models mimicking inflammatory bowel disease. Inflamm Bowel Dis 13: 325330 .

14. Chen J, Huang XF (2009) The signal pathways in azoxymethane-induced colon cancer and preventive implications. Cancer Biol Ther 8: 1313-1317.

15. Suzuki R, Kohno H, Sugie S, Nakagama H, Tanaka T (2006) Strain differences in the susceptibility to azoxymethane and dextran sodium sulfate-induced colon carcinogenesis in mice. Carcinogenesis 27: 162-169. 
Citation: Venning FA, Claesson MH, Kissow H (2013) The Carcinogenic Agent Azoxymethane (AOM) Enhances Early Inflammation-induced Colon Crypt Pathology. J Cancer Sci Ther 5: 377-383. doi:10.4172/1948-5956.1000229

16. Tanaka T, Kohno $H$, Suzuki R, Yamada $Y$, Sugie $S$, et al. (2003) A novel inflammation-related mouse colon carcinogenesis model induced by azoxymethane and dextran sodium sulfate. Cancer Sci 94: 965-973.

17. Kjellev S, Lundsgaard D, Poulsen SS, Markholst H (2006) Reconstitution of Scid mice with CD4+CD25- T cells leads to rapid colitis: an improved model for pharmacologic testing. Int Immunopharmacol 6: 1341-1354

18. Bird RP (1987) Observation and quantification of aberrant crypts in the murine colon treated with a colon carcinogen: preliminary findings. Cancer Lett 37 : 147-151.

19. Kissow H, Hartmann B, Holst JJ, Viby NE, Hansen LS, et al. (2012) Glucagonlike peptide-1 (GLP-1) receptor agonism or DPP-4 inhibition does not accelerate neoplasia in carcinogen treated mice. Regul Pept 179: 91-100.

20. Ramos-Vara JA (2005) Technical aspects of immunohistochemistry. Vet Patho 42: 405-426.

21. Asting AG, Carén H, Andersson M, Lönnroth C, Lagerstedt $K$, et al. (2011) COX-2 gene expression in colon cancer tissue related to regulating factors and promoter methylation status. BMC Cancer 11: 238.

22. Corpet DE, Taché S (2002) Most effective colon cancer chemopreventive agents in rats: a systematic review of aberrant crypt foci and tumor data, ranked by potency. Nutr Cancer 43: 1-21

23. Seike K, Koda K, Oda K, Kosugi C, Shimizu K, et al. (2006) Assessment of rectal aberrant crypt foci by standard chromoscopy and its predictive value for colonic advanced neoplasms. Am J Gastroenterol 101: 1362-1369.

24. Blunt T, Gell D, Fox M, Taccioli GE, Lehmann AR, et al. (1996) Identification of a nonsense mutation in the carboxyl-terminal region of DNA-dependent protein kinase catalytic subunit in the scid mouse. Proc Natl Acad Sci U S A 93: 1028510290.

25. Smith GC, Jackson SP (1999) The DNA-dependent protein kinase. Genes Dev 13: $916-934$

26. McCune JM (1991) SCID mice as immune system models. Curr Opin Immuno 3: $224-228$.

27. Ochiai M, Ubagai T, Kawamori T, Imai H, Sugimura T, et al. (2001) High susceptibility of Scid mice to colon carcinogenesis induced by azoxymethane indicates a possible caretaker role for DNA-dependent protein kinase. Carcinogenesis 22: 1551-1555.

28. Anderson JC, Swede H, Rustagi T, Protiva P, Pleau D, et al. (2012) Aberrant crypt foci as predictors of colorectal neoplasia on repeat colonoscopy. Cancer Causes Control 23: 355-361. 